\title{
Rapid Detection of Tetracycline Residues in Chicken
}

\author{
Shraddha Patrabansh, Niranjan Parajuli, Vinay Kumar Jha* \\ Central Department of Chemistry, Tribhuvan University, Kirtipur, Kathmandu, Nepal
}

\author{
Article Information \\ Received: 02 January 2020 \\ Revised version received: 25 February 2020 \\ Accepted: 10 March 2020 \\ Published: 29 March 2020

\section{Cite this article as:} \\ S. Patrabansh et al. (2020) Int. J. Appl. Sci. Biotechnol. \\ Vol 8(1): 14-20. DOI: $\underline{10.3126 / i j a s b t . v 8 i 1.27201}$

\section{*Corresponding author} \\ Vinay Kumar Jha, \\ Central Department of Chemistry, Tribhuvan University, \\ Kirtipur, Kathmandu, Nepal \\ Email: vinayj2@yahoo.com \\ Tel. No.: +977-1-4335572 (Res.), +977-1-4332034 (O.) \\ Peer reviewed under authority of IJASBT \\ (C) 2020 International Journal of Applied Sciences and \\ Biotechnology
}

\section{OPEN $O$ ACCESS}

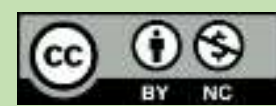

This is an open access article \& it is licensed under a Creative Commons Attribution Non-Commercial 4.0 International (https://creativecommons.org/licenses/by-nc/4.0/)

Keywords: Antibiotic residues; Chicken; HPLC-UV; Quicking Tetracycline rapid Test kit; Tetracycline

\begin{abstract}
Antibiotic residues in meat are a serious public health concern as a result of its harmful effects on consumer health. This study aimed at estimating the residues levels of commonly used antibiotics (Tetracycline) in chicken samples using two analytical methods; Rapid Screening Kit and HPLC.

Twenty chicken samples were collected from various meat shops of Kathmandu valley. Qualitative and semi-quantitative analyses with Quicking Tetracycline rapid Test kit, which detect tetracycline above $100 \mathrm{ppb}$ in tissue revealed that two samples were positive for antibiotic residues in the chicken for tetracycline. The concentration of residues of tetracycline, which were positive in rapid test kit was quantified by high-performance liquid chromatography equipped with a UV detector (HPLC-UV). One muscle sample and one liver sample of chicken have found the concentration of tetracycline as 229 and $339 \mu \mathrm{g} / \mathrm{kg}$, respectively while chlortetracycline was not detected. This result confirmed widespread misuses of antibiotics especially tetracycline in farm and lack of application of recommended withdrawal times. Antimicrobial assay of chicken extracts was also performed by using the disc-diffusion agar method. The plates were incubated overnight at $37{ }^{\circ} \mathrm{C}$ for $18-24$ hours, but microbial growth was not observed in all the samples tested.
\end{abstract}

\section{Introduction}

A broiler is grown commercially for meat as they grow rapidly and the financial investment is low (Saxena $e t$ al., 2014). As the demand for meat is increasing day by day, several agents have been using for growth promotion and treatment of the product. When antibiotics are given to animals, part of the antibiotic and its by-products will result in the flesh and the product (honey, milk, eggs, etc.) of the animal. However, after some days, the level gradually declines because of metabolic degradation. The antibiotic withdrawal period is the time that passes between the last doses of an antibiotic given to the animal and the time when the level of antibiotic residues, i.e. animal's flesh or products, falls below the maximum residues limit (MRL). Until the withdrawal period has expired, the animal or its products are not suitable for human consumption (Farm Animals, 2012).

Antibiotics are invaluable chemical probes, mostly produced by soil microbes that inhibit the growth of microorganisms or destroy them. Veterinary medicine utilizes antibiotics as a therapeutic, prophylactic, and growth-promoting agent as well as nutritive purposes in poultry and livestock production. They can be administered either in feed, in drinking water, or by injection, and some 
use "cocktails", i.e. mixtures of small amounts of several substances. Residues or their metabolites may cause adverse effects after its consumption to the humans. Carcinogenicity, mutagenicity, bone marrow toxicity (Chloramphenicol) and allergy (Penicillin) are some of the hazards of consumption of antibiotics residues in food (Nisha, 2008; Yu et al., 2011). Bacterial resistance is another serious issue due to the disruption of intestinal microflora (Singh et al., 2014).

Tetracyclines are a family of antimicrobials that inhibit protein synthesis by preventing attachment of aminoacyltRNA to the ribosomal acceptor (A) site (Chopra et al., 2001). Tetracyclines consist of a common four-ring structure to which a variety of side chains are attached (Prescott et al., 2002). Because of their broad-spectrum activity and low cost, tetracycline (TC) including oxytetracycline (OTC), chlortetracycline (CTC), and doxycycline (DC) are widely used in animals for both prevention, treatment and as feed, additives to promote growth ( Abbasi et al., 2011).

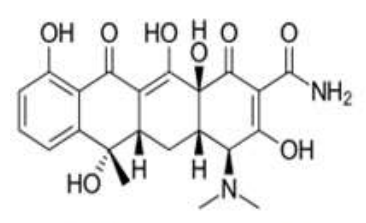

Tetracycline

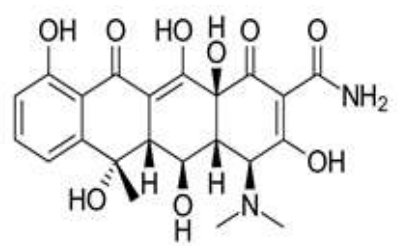

Oxytetracycline

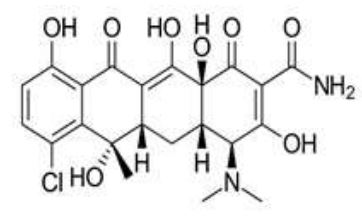

Chlortetracycline

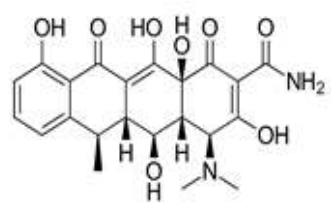

Doxycycline
Maximum residues limits (MRLs) for TCs were recommended by many agencies. The joint FAO/WHO Expert Committee on Food Additives recommended 200 and $600 \mu \mathrm{g} / \mathrm{kg}$ for chicken muscle and liver, respectively, expressed as the sum of TCs (FAO/WHO report). In addition, the EU had set the MRLs of TCs to be 100 and $300 \mu \mathrm{g} / \mathrm{kg}$ for chicken muscle and liver, respectively (Commission Regulation (EU) No 37/2010, 2009).

Antibiotic residues are detected by chemical, biological and immunological methods. Detection methods can be classified into qualitative, semi-quantitative, and quantitative. In this study, tetracycline rapid test kit was used to detect the presence of tetracycline residues in animal tissue, and HPLC was used to evaluate tetracycline residues in positive samples resulted from a rapid test kit. Antimicrobial assay of chicken extracts was performed through the disc diffusion agar method.

\section{Materials and Methods}

\section{Sample Collection and Preservation (Procedure)}

Twenty chicken samples were collected randomly from different meat shops of Kathmandu valley. In most of the previous studies, the antibiotics residues were evaluated in muscle, liver, and kidney (Naeem et al., 2006). Hence chicken muscle and liver as samples were purchased and fat tissues were removed. Samples were minced and packed in good quality polybags, sealed and kept in a deep freezer at $-20{ }^{\circ} \mathrm{C}$ till tests were done.

\section{Evaluation of Antibiotic Residues}

In this study, two methods were used simultaneously for the determination of antibiotic residues in chicken.

A. Qualitative and semi-quantitative analysis of antibiotic residues by Quicking Tetracycline Rapid Test kit (obtained from Quicking Biotech Co., Ltd. Shanghai, China).

B. Quantitative analysis of meat samples by HPLC equipped with a UV Detector (HPLC-UV).

\section{A. Tetracycline Rapid Test Kit}

\section{Principle of the assay}

This test is based on competitive lateral flow immunochromatographic assay. The TC-conjugate in the test zone will capture the immune-gold (colloid gold-TC antibody conjugate) when there is very little dissociative TC in the samples. A visible red test band specifies a negative result when the control line ( $\mathrm{C}$ zone) shows that the card is valid. The test band ( $\mathrm{T}$ zone) will be not visible if $\mathrm{TC}$ is present in a concentration of $100 \mathrm{ppb}$ and above, which explains a positive result. The protocol of the test kit is to be followed as per the instructions are given in the manual provided by the Quicking Biotech Co. Ltd (Tetracycline Rapid Test Kit (tissue), 2010).

\section{a) Equipment for Rapid test kit:}

Quicking Tetracycline Rapid Test Kit, Homogenizer, Water bath $\left(80^{\circ} \mathrm{C}\right)$, Centrifuge, Balance were used.

\section{b) Test procedure}

The sample was homogenized at 10,000 rpm for $1 \mathrm{~min} .4 \mathrm{~g}$ of sample was weighed into $15 \mathrm{~mL}$ centrifugal tube, and 0.5 $\mathrm{mL}$ of assay buffer was added, and the lid was covered tightly. The tube was put into the water bath $\left(80^{\circ} \mathrm{C}\right)$ for 10 min, and the extract was taken out as much into $1.5 \mathrm{~mL}$ of the centrifugal tube.

Then it was centrifuged at $4000 \mathrm{rpm}$ for $1 \mathrm{~min}$ to make extract clear. $0.2 \mathrm{~mL}$ of the extract was sucked into microwell with a pipette. Repeatedly sucked, and the sample was extruded until all red reagents were completely dissolved and waited for $1 \mathrm{~min}$. Gradually three drops of sample extraction were dripped into the sample hole " $\mathrm{S}$ " of the Cassette horizontally. The result was interpreted in 5-10 
min. The result after $10 \mathrm{~min}$. was only considered as reference.

\section{B. Quantitative estimation from High-Performance Liquid Chromatography}

Tetracycline was extracted from poultry tissue with Mcllvaine-EDTA buffer having $\mathrm{pH}$ 4. The filtered extract was cleaned up and enriched with solid-phase extraction (SPE). Tetracycline was separated by liquid chromatography using a C18 column and measured with a UV detector at $350 \mathrm{~nm}$. The signal of the sample was compared with those from standard solutions of Tetracycline, and Chlortetracycline. Concentration in the sample was calculated with the help of the formula reported (Macneil et al. 1996).

\section{Sample processing equipment and Devices and Consumables for HPLC}

i. Apparatus: The HPLC equipped with a UV detector was used. Analytical balance, pH meter, Centrifuge, Sonicator, Vortex mixture, Shaker, SPE aperture, C18 SPE cartridges, and Nitrogen evaporation device were used for sample preparation.

ii. Chemicals and reagents: Standard NIST traceable CRM of tetracycline \& chlorotetracycline were used. Methanol, water, and formic acid were of HPLC grade. $\mathrm{Na}_{2} \mathrm{HPO}_{4}$, EDTA dihydrate, citric acid monohydrate, and oxalic acid dehydrate were of analytical grade.

\section{b) Extraction Mixture}

Mcllvaine Buffer: $\mathrm{Na}_{2} \mathrm{HPO}_{4}(28.4 \mathrm{~g})$ was dissolved in $1 \mathrm{~L}$ volumetric flask with water, and $21 \mathrm{~g}$ citric acid monohydrate was dissolved in 1 Lvolumetric flask with water. The citric acid solution $(500 \mathrm{~mL})$ and $312.5 \mathrm{~mL}$ $\mathrm{Na}_{2} \mathrm{HPO}_{4}$ solution were combined, and $\mathrm{pH}$ was adjusted to $4 \pm 0.05$ by using either $0.1 \mathrm{M} \mathrm{HCl}$ or $0.1 \mathrm{M} \mathrm{NaOH}$ solution.

Na2 EDTA-Mcllvaine Buffer: $\mathrm{Na}_{2}$ EDTA $2 \mathrm{H}_{2} \mathrm{O}(30.25 \mathrm{~g})$ was added to the previously prepared Mcllvaine buffer solution and dissolved.

Methanolic oxalic acid: Oxalic acid (1.26 g) was weighed and dehydrated into $1 \mathrm{~L}$ volumetric flask and dissolved in methanol, and volume was made up to the mark with methanol.

\section{c) Preparation of Mobile Phase}

Mobile phase A: Oxalic acid (1.26 g) was dehydrated into a $1 \mathrm{~L}$ volumetric flask, dissolved in water and made volume up to the mark with water. It was filtered and sonicated.

Mobile phase B: Acetonitrile $(600 \mathrm{~mL})$ and $200 \mathrm{~mL}$ methanol were added in a beaker and sonicated.

\section{d) Standard Solutions}

Stock standard solutions of each standard (tetracycline and chlortetracycline) was prepared by dissolving $10 \mathrm{mg}$ of the compound in $10 \mathrm{~mL}$ methanol to obtain a final concentration of $1000 \mathrm{ppm}$. Stock standard solutions were diluted with methanol to give a series of working standard solutions that were prepared frequently.

\section{e) Sample Extraction}

Sample $(5 \mathrm{~g})$ was taken in a $50 \mathrm{~mL}$ centrifuge tube, and 20 $\mathrm{mL}$ extraction buffer was added. The mixture was then closed with a screw cap and vortexed for 30 seconds followed by 10 minutes shaking in a mechanical shaker. The mixture was centrifuged at $6000 \mathrm{rpm}$ for 10 minutes. The upper layer of the supernatant solution was taken in a New $50 \mathrm{~mL}$ centrifuge tube. The extraction steps were repeated twice with $15-$ and $10-\mathrm{mL}$ extraction buffer respectively. Supernatants were collected at each extraction step, and all supernatants were mixed into the same centrifuge tube, then centrifuged for $15 \mathrm{~min}$ at $6000 \mathrm{rpm}$. A filter paper was placed in a funnel and moistened with $1 \mathrm{~mL}$ extraction Buffer. The supernatants were filtered. The centrifuge tube was washed and filtered with $4 \mathrm{~mL}$ of extraction buffer. Centrifuged for $15 \mathrm{~min}$ at $6000 \mathrm{rpm}$ and proceed with SPE.

\section{f) SPE Cleanup}

Conditioning: The SPE cartridges conditioned with3 times $2 \mathrm{~mL} \mathrm{MeOH}$ under gravity flow and discarded the eluate. Next, three times $2 \mathrm{~mL}$ of water was added with gravity flow and discarded the eluate (Precaution: cartridges should not be run in dry).

Loading: The extract was added stepwise into the cartridge with a gentle vacuum (speed approx. 1-2 drop/s). Cartridges should not run dry during loading.

Drying: Cartridges was dried for 2 min using a vacuum.

Elute: The contents of cartridges were eluted using 3 times $2 \mathrm{~mL}$ Methanolic oxalic acid into $10 \mathrm{~mL}$ volumetric flask.

After elution, volume was made up to the mark with water and shaken. Filtered through $0.45 \mu \mathrm{m}$ syringe membrane; filtered into HPLC vial and injected into the HPLC system.

\section{g) Detection and Quantification}

The separated TCs were detected with Ultraviolet detector, and the quantification was integrated by chromatographic software interfaced to a personal computer and using the regression equation.

\section{Antimicrobial Activity}

a. Antimicrobial activity assays: Antimicrobial assay of chicken extracts was performed by using Nutrient Agar(NA) Medium. Nutrient Broth is convenient, as most bacteria grow in this type of medium.

b. Preparation of extracts: Meat sample was homogenized at 10,000 rpm for $1 \mathrm{~min} .4 \mathrm{~g}$ of it was weighed into $15 \mathrm{~mL}$ centrifugal tube, and $500 \mu \mathrm{L}$ of $50 \mathrm{mM}$ Tris buffer ( $\mathrm{pH}$ 8) was added. Then centrifuged at $12,000 \mathrm{rpm}$ for $1 \mathrm{~min}$ to make the extract clear. The supernatant was used for the assays.

c. Screening and Evaluation of Antimicrobial Activity: All instruments, solutions, and media before using them for plating procedures were sterilized, and workspace was set up in a Biosafety cabinet. 
The supernatant was picked up and spread over the surface of the NA agar medium using a rapid, smooth, back-andforth motion by a metal loop. The plate was inverted and set down back into the lid. The plates were incubated overnight at $37{ }^{\circ} \mathrm{C}$. After proper incubation (18-24 hours), the plates were observed for bacterial growth.

\section{Results and Discussion}

\section{Antibiotic Residues in Chicken Analyzed by Rapid Screening Test Kits}

Out of 20 meat samples tested by Quicking Tetracycline Rapid Test Kit, two samples were found positive for the tetracycline residues (shown in Fig. 1). Semi-quantitative tests revealed that all positive samples contained tetracycline residues in the concentration of $100 \mathrm{ppb}$ and above (Table 1). The results were interpreted based on the clear band appearing in the $\mathrm{C}$ and $\mathrm{T}$ zone.

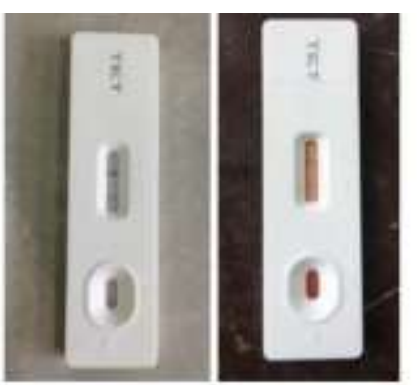

(a)

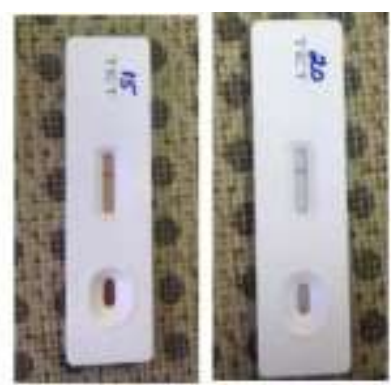

(b)
Fig. 1: Samples showing (a) the negative and (b) positive results for Tetracycline residues.

In Fig. 1 (a) the result showed that tetracycline residues were absent in a meat sample due to the appearance of the clear band in both $\mathrm{C}$ and $\mathrm{T}$ zone while in Fig. 1 (b) the result showed that tetracycline residues were present in the meat sample as the clear band appeared in only $\mathrm{C}$ zone.

\section{Antibiotic Residues Analyzed by HPLC Method}

Wavelength Screening: In this study, $350 \mathrm{~nm}$ was used for the tetracycline absorption wavelength with a UV detector.

Linearity and Range: Standard tetracycline solution of five different concentrations ranging from 50 to $1000 \mathrm{ppb}$ was analyzed. The calibration curve was established according to the peak area and the concentration of the Tetracycline in standard solutions. The result showed a linear relationship in the concentration range of 50 to 1000 ppb (shown in Fig. 2).

Table 1: Tetracycline residues in chicken samples from Kathmandu valley analyzed by Quicking Tetracycline Rapid Test Kit.

\begin{tabular}{|l|l|l|l|}
\hline $\begin{array}{l}\text { S. } \\
\text { No. }\end{array}$ & $\begin{array}{l}\text { Sample } \\
\text { Type }\end{array}$ & Location & Tetracycline \\
\hline 1 & Muscle & Sanepa & Negative \\
\hline 2 & Liver & Sanepa & Negative \\
\hline 3 & Muscle & Kalimati & Negative \\
\hline 4 & Muscle & Kalanki & Negative \\
\hline 5 & Muscle & Lagankhel & Negative \\
\hline 6 & Muscle & Balaju & Negative \\
\hline 7 & Muscle & Bhaktapur & Negative \\
\hline 8 & Muscle & ChappalKarkhana & Negative \\
\hline 9 & Muscle & Basbari & Negative \\
\hline 10 & Muscle & Maharajgunj & Negative \\
\hline 11 & Muscle & Aakasedhara & Negative \\
\hline 12 & Liver & Teku & Negative \\
\hline 13 & Muscle & Kirtipur & Negative \\
\hline 14 & Liver & Godawari & Negative \\
\hline $\mathbf{1 5}$ & Liver & Khokana & Positive \\
\hline 16 & Muscle & Khokana & Negative \\
\hline 17 & Liver & Harisiddhi & Negative \\
\hline 18 & Muscle & Patan & Negative \\
\hline 19 & Muscle & Samakhusi & Negative \\
\hline $\mathbf{2 0}$ & Muscle & Tokha & Positive \\
\hline
\end{tabular}

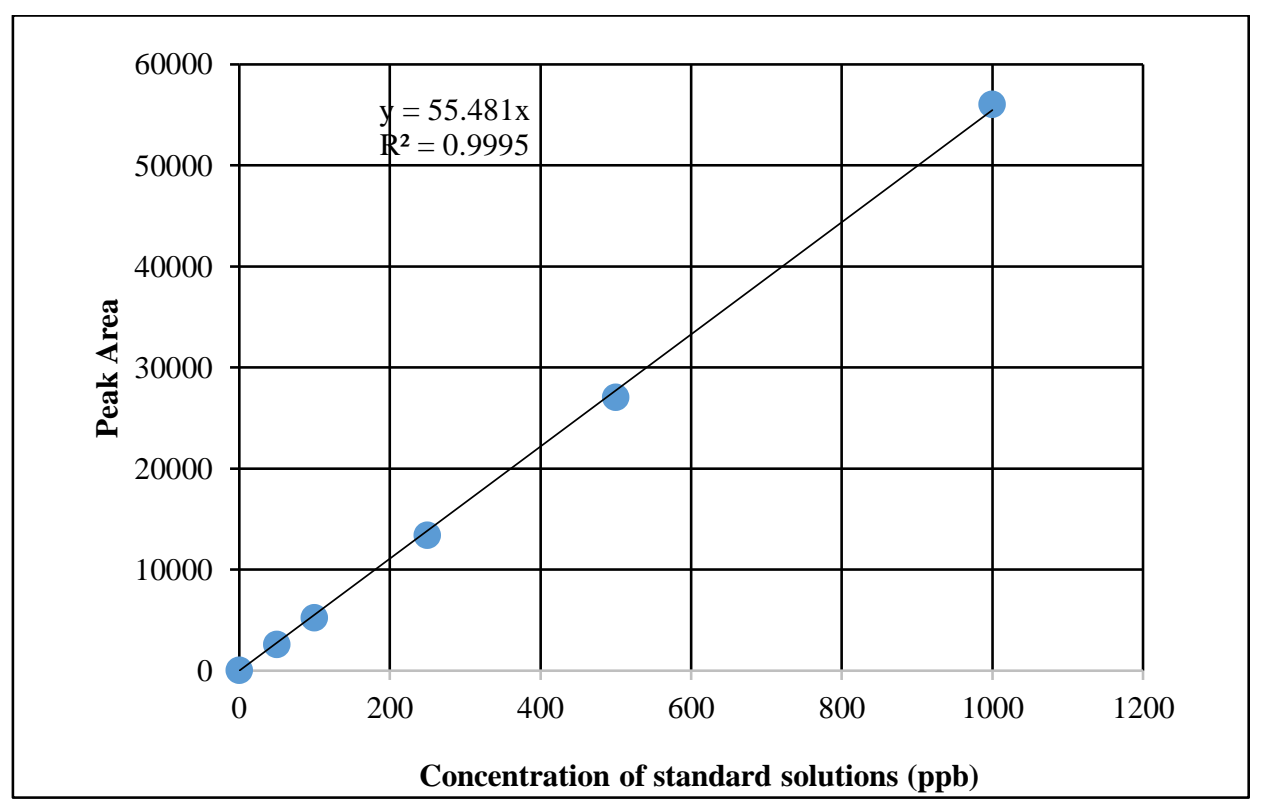

Fig. 2: Calibration curve for the standard Tetracycline solutions 


\section{Sensitivity}

\section{Limit of Detection}

The limit of detection is used to decide whether an analyte is present, while the limit of quantification is used to decide whether the concentration of an analyte can be reliably determined (Michaele et al., 1999). Mathematically, it can be expressed as:

Limit of detection

$=\frac{3 \times \text { standard deviation of intercept }}{\text { slope of calibration line }}$

\section{Limit of Quantification}

Limit of quantificationis the lowest concentration of an analyte in a sample that can be determined with acceptable precision and accuracy under the stated conditions of the test (Shrivastava et al., 2011). Mathematically,

Limit of quantification

$=\frac{10 \times \text { standard deviation of intercept }}{\text { slope of calibration line }}$

The limit of detection and limit of quantification were 30 $\mathrm{ppb}$ and $100 \mathrm{ppb}$, respectively. The regression equation for tetracycline was:

$y=55.481 x \quad \cdots \cdots \cdots$

The coefficient of determination $\left(\mathrm{R}^{2}\right)$ of the line was 0.9995 .

All the results obtained from this experiment are presented in the following assay validation Table 2.

Table 2: Assay validation Table

\begin{tabular}{|l|l|l|}
\hline $\begin{array}{l}\text { S. } \\
\text { No. }\end{array}$ & Parameter & Value \\
\hline 1 & Accuracy (mean \pm s.d) (in \%) & $\begin{array}{l}96.278 \pm \\
3.212\end{array}$ \\
\hline 2 & slope (a) & 55.481 \\
\hline 3 & Linearity range (in ppb) & $50: 1000$ \\
\hline 4 & Coefficient of determination $\left(\mathrm{R}^{2}\right)$ & 0.9995 \\
\hline 5 & The standard error of intercept & 285.005 \\
\hline 6 & $\begin{array}{l}\text { The standard deviation of the } \\
\text { intercept }\end{array}$ & 635.562 \\
\hline 7 & Limit of detection (in ppb) & 30 \\
\hline 8 & Limit of quantification (in $\mathrm{ppb})$ & 100 \\
\hline
\end{tabular}

\section{Results of Tetracycline Residues in Chicken Sample}

Tetracycline residues in the chicken samples that were sold in the market of Kathmandu and were positive inQuicking Tetracycline Rapid Test Kitwere analyzed by using HPLC coupled to ultraviolet detection. Peak identification of the sample was based on the comparison with a retention time of standard compounds peaks. The external standard method was used for quantification using calibration curves fitted by linear regression analysis. The chromatograms obtained from the highest standard solution of Tetracycline and samples of meat are shown in Fig. 3 respectively. [In all the chromatograms the $\mathrm{X}$-axis is retention time in minutes and $\mathrm{Y}$-axis is Detector response in milliAbsorbance Units (mAU)].

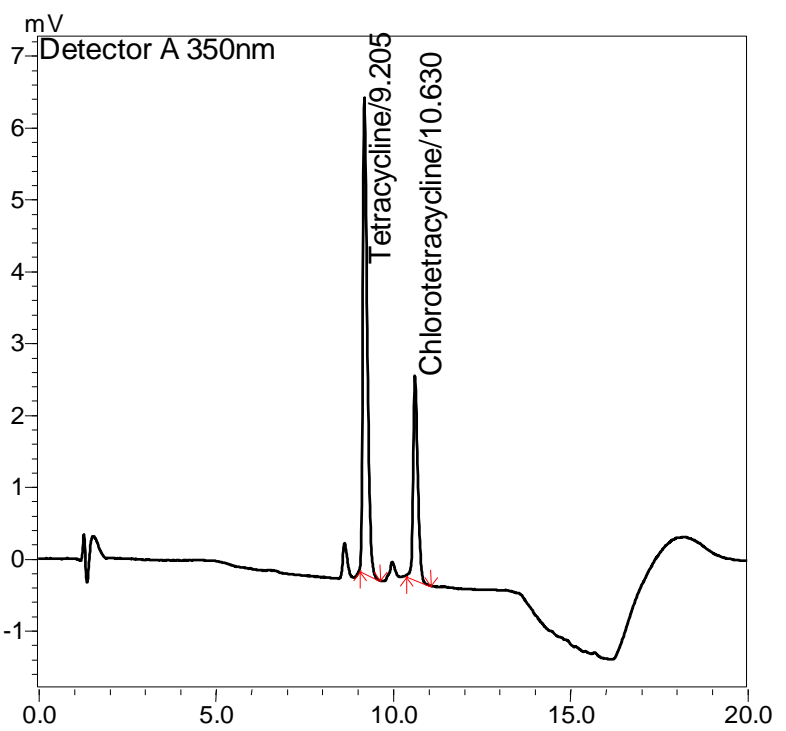

Fig. 3: HPLC Chromatogram of 1000 ppb STD (Highest STD)

From the chromatogram of standard Tetracycline solution, it has been shown that the retention time for Tetracycline was at 9.205 minutes. This retention time was used for the identification of the peak of interest in the chromatogram of the sample. The retention time for chlortetracycline was 10.630 minutes.

Tetracycline residues were detected in chicken samples, which were identified based on retention time, i.e. 9.205 minutes, while no detection level signal was seen for the retention time 10.630 minutes and hence tetracycline's analytical signal was only detected and is shown in Fig. 4.

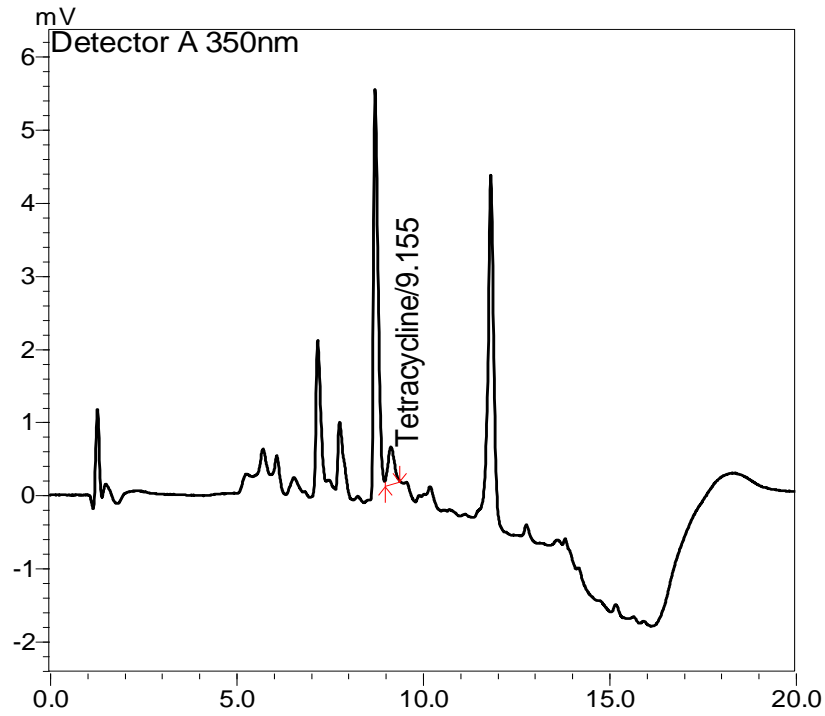

Fig. 4: HPLC Chromatogram of chicken liver (171.97 ppb in aliquot). 
By knowing the value of the analytical signal of the unknown from the HPLC, the concentration of tetracycline in HPLC was calculated by using the following formula:

Concentration of Tetracycline in HPLC =

$\frac{\text { Analytical signal - intercept }}{\text { slope }} \quad \ldots \ldots$

The concentration of tetracycline residues in aliquot (HPLC) of liver and muscle samples was found to be 171.97 ppb and $115.08 \mathrm{ppb}$, respectively.

Then the concentration of tetracycline obtained in the sample was calculated by using the following formula:

Conc. of Tetracycline in poultry tissue $(\mu \mathrm{g} / \mathrm{kg})$

$=\frac{H P L C \text { reading }(p p b) \times \text { Final volume in } H P L C}{\text { weight taken }(g)} \ldots$

The quantity of tetracycline in liver and muscle was 339 and $229 \mu \mathrm{g} / \mathrm{kg}$, respectively, and is shown in Fig. 5.

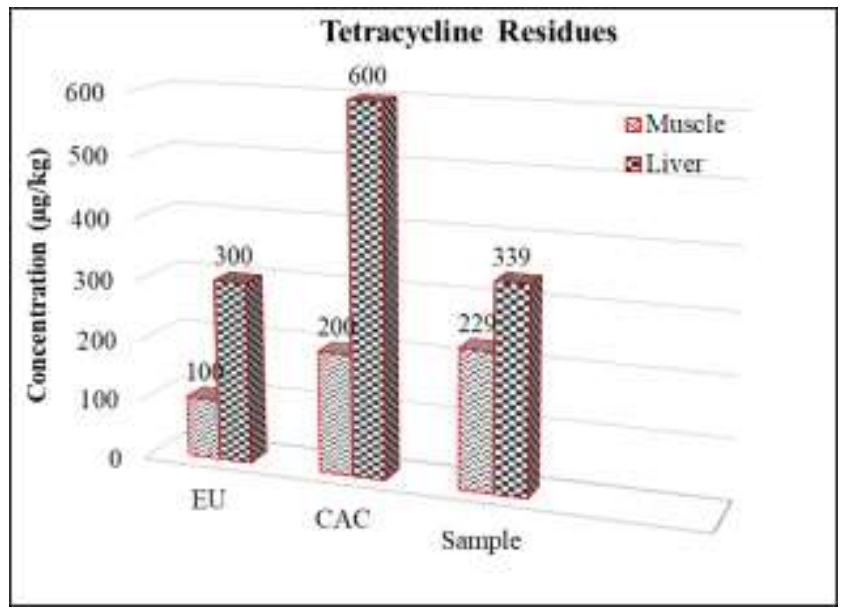

Fig. 5: Concentration of Tetracycline residues [*EU and CAC are MRL values of Tetracycline Residues according to European Union and Codex Alimentarius Commission respectively, while a sample is tetracycline residues of the present studied sample.]

The quantitative value of tetracycline residue in the muscle of the studied sample was found higher than the MRL value of both EU and CAC while that of the liver's tetracycline residue value was higher than EU's but within CAC's maximum residue limit.

This preliminary research was reported the presence of antibiotic residues in poultry samples coming Kathmandu. Among the primary screening of the 20 samples of chicken meat samples, 2 samples were found contaminated with relatively high tetracycline residue. The level of antibiotic consumption in the Nepalese veterinary medicine should be evaluated.

\section{Antimicrobial Screening}

Antimicrobial assay of chicken extracts was performed by using Nutrient Broth Agar Medium. The plates were incubated overnight at $37^{\circ} \mathrm{C}$. After proper incubation (1824 hours), bacterial growth was not noticed in all the samples (data not shown).

\section{Conclusion}

Residues of antibiotics were detected in the chicken of Kathmandu by Rapid screening test kit and HPLC. Results of the tetracycline Rapid Screening Test Kit showed that two samples were positive for tetracycline residues. The positive samples were further quantified by HPLC. The liver and muscle samples were found to contain 339 and 229 $\mu \mathrm{g} / \mathrm{kg}$ tetracycline residues, respectively. Chlortetracycline was not detected in both samples.

This study urges the need of an intervention to decrease the level of antibiotics residues in chicken samples through launching educational and awareness programs on the prudent use of antibiotics in animal husbandry. The studies should be conducted to screen a larger sample collected from different farms located in different regions in the country. The subsequent researches would need focus on various parts of Nepal to complete the available data and to provide a complete view of antibiotic residues in chickens for entire country.

\section{Author's Contribution}

VK Jha designed the research plan; S Patrabansh performed experimental works, collected the required data \& prepared the manuscript. S Patrabansh, N Parajuli, and VK Jha analysed the data; critically revised and finalized the manuscript. Final form of manuscript was approved by all authors.

\section{Conflict of Interest}

The authors declare that there is no conflict of interest with present publication.

\section{Acknowledgements}

One of the authors (S. Patrabansh) gratefully acknowledgesthe University Grants Commission, Nepal for providing a UGC Masters Research Support (Award No.: MRS/74_75/S\&T-36) for this study. We are also thankful to the Department of Food Technology and Quality Control (Government of Nepal), Central Department of Biotechnology, Tribhuvan University for providing laboratory facilities.

\section{References}

Abbasi MM, Babaei H and Ansarin M (2011) Simultaneous determination of tetracyclines residues in bovine milk samples by solid phase extraction and HPLC-FL method. Advanced Pharmaceutical Bulletin. 1(1): 34. DOI: 10.5681/apb.2011.005

Chopra I, and Roberts M (2001) Tetracycline antibiotics: mode of action, applications, molecular biology, and epidemiology of bacterial resistance. Microbiol Mol Biol Rev 65(2): 232260. DOI: 0.1128/MMBR.65.2.232-260.2001 
Commission Regulation (EU) No. 37/2010 of 22 December 2009 on pharmacologically active substances and their classification regarding maximum residues limits in foodstuffs of animal origin. (2010). (470): 72.

FAO/WHO report (http://www.fao.org/fao-whocodexalimentarius/en/ Retrieved April 17, 2019)

Farm Animals: Antibiotic Withdrawal Period and How It Affects Your Health «FarmersJoint Blog. (n.d.). (http://www.farmersjoint.com/blog/livestockfarming/farm-animals-antibiotic-withdrawal-periodaffects-health Retrieved April 17, 2019).

Macneil JD, Martz VK, Korsrud GO, Salisbury CD, Oka H, Epstein RL, and Barnes CJ (1996) Chlortetracycline, oxytetracycline, and tetracycline in edible animal tissues, liquid chromatographic method: collaborative study. $J$ AOAC Int 79(2): 405-417.

Naeem M, Khan K, and Rafiq S (2006) Determination of residues of quinolones in poultry products by high pressure liquid chromatography. J Appl Sci 6: 373-379. DOI: $\underline{10.3923 / j a s .2006 .373 .379}$

Nisha AR (2008) Antibiotic Residues - A Global Health Hazard. Veterinary World, 1(12), 375-377. DOI: $\underline{10.5455 / \text { vetworld.2008.375-377 }}$

Prescott LM, Harley JP, and Klein DA (2002).Microbiology (5thInt'l Ed.). (https://trove.nla.gov.au/version/35077377; Retrieved April 15, 2019).
Saxena P, and Sahu R (2014). CSE Study: Antibiotics in Chicken Meat (at Centre for Science and Environment, Pollution Monitoring Laboratory, New Delhi, India). 1-36.

Shrivastava A, and Gupta V (2011). Methods for the determination of limit of detection and limit of quantitation of the analytical methods. Chron Young Sci 2(1):21-25. DOI: $\underline{10.4103 / 2229-5186.79345}$

Singh S, Shukla S, Tandia N, Kumar N, and Paliwal R (2014). Antibiotic Residues: A Global Challenge. Pharma Science Monitor 5: 184-197.

Tetracycline Rapid Test Kit (tissue) - Buy Tetracycline, Food Safety Testing Kit, Tetracycline Residues in Meat Product on Alibaba.com. (2010). (www.alibaba.com/productdetail/Tetracycline-Rapid-Test-Kit-Tissue.html Retrieved April 22, 2019).

Yu H, Tao Y, Chen D, Wang Y and Yuan Z (2011). Development of an HPLC-UV method for the simultaneous determination of tetracyclines in muscle and liver of porcine, chicken and bovine with accelerated solvent extraction. Food Chem 124: 1131-1138. DOI: 10.1016/j.foodchem.2010.07.024

Zorn ME, Gibbons RD, and Sonzogni WC (1999). Evaluation of Approximate Methods for Calculating the Limit of Detection and Limit of Quantification. Environ SciTechnol, 33: 2291-2295. DOI: 10.1021/es981133b 